\title{
Fundamentals of Optomechanics
}

: IIn damenta|s at

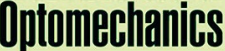
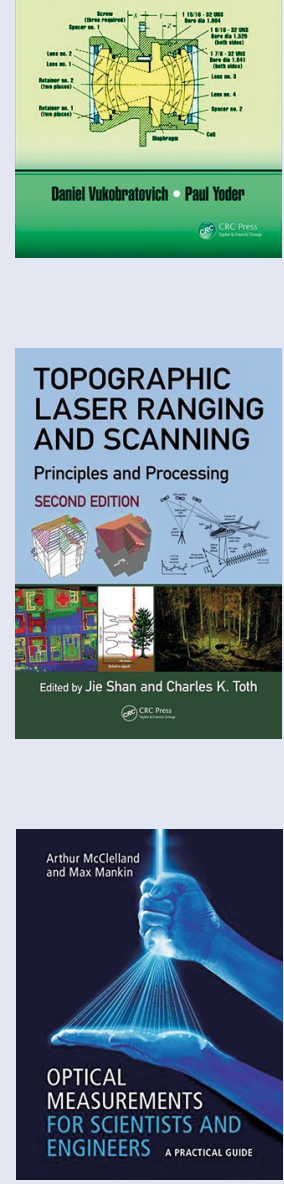

CRC PRESS: 2018. 448PP. £100.00.

Organized into 11 chapters, this book aims to provide the fundamentals of optomechanics. The first few chapters give details on the design process, material selection and the principles of kinematic design. Descriptions on mounting windows, mounting individual lenses, mounting multiple lens assemblies and techniques for mounting prisms are also covered. The last few chapters focus on the factors affecting mirror performance, design and mounting of small mirrors and metallic mirrors. Also included are homework problems and a solution manual.

\section{Topographic Laser Ranging and Scanning}

Edited by Jie Shan and Charles K. Toth

CRC PRESS: 2018. 638PP. E144.00.

Composed of 19 chapters, this title discusses topographic light detection and ranging (LIDAR) principles, systems, and data-acquisition and data-processing techniques. It covers the fundamentals of ranging and scanning, and includes a contemporary analysis of terrestrial, airborne and spaceborne LIDAR systems. Data collection in terms of waveforms and their properties, accuracy, quality control and data management, and LIDAR data processing and information extraction are discussed. Filtering algorithms for digital-terrain-model generation and for integration of LIDAR and photogrammetric data are also included. Last, but not least, the book provides examples of the practical and commercial achievements and opportunities of LIDAR systems.

\section{Optical Measurements for Scientists and Engineers}

By Arthur McClelland and Max Mankin

CAMBRIDGE UNIV. PRESS: 2018. 320PP. E69.99.

Starting from the basics, this book introduces light and its properties, such as reflection, total internal reflection, polarization, absorption, scattering, emission, birefringence and interference. It then introduces laser sources and other common optical components used in the lab. Chapter 3 is devoted to different types of optical spectroscopy, such as absorption spectroscopy, fluorescence spectroscopy, photoluminescence spectroscopy, Fourier transform infrared spectroscopy, Raman spectroscopy and laser-induced breakdown spectroscopy. Chapter 4 details different types of imaging techniques and microscopy, such as bright- and dark-field imaging, phase contrast imaging, confocal microscopy, light-sheet microscopy, multiphoton microscopy and super-resolution microscopy. With practical lab tips throughout, this text provides step-by-step instructions on how to select the best technique for a particular application, how to set up and customize new optical systems, and how to analyse optical data.

\section{Confocal Raman Microscopy}

Edited by Jan Toporski, Thomas Dieing and Olaf Hollricher SPRINGER: 2018. 622PP. £119.99.

This book provides an overview of the physical, technical and scientific aspects related to the widely used analytical method of confocal Raman microscopy. This second edition extends background information and gives insights into the integration of confocal Raman microscopy with other methods. The topics covered include software requirements and data analysis in confocal Raman microscopy, resolution and performance of three-dimensional confocal Raman imaging systems, nano-spectroscopy of individual carbon nanotubes and isolated graphene sheets, characterization of graphene by confocal Raman spectroscopy, and low-frequency Raman scattering of two-dimensional materials beyond graphene. Applications of confocal Raman microscopy in various fields, such as mineralogy and petrography, archaeology, intracellular drug delivery and cancer diagnosis, are also described.

Published online: 26 April 2018

https://doi.org/10.1038/s41566-018-0165-9 